\title{
A cadaveric study of ultrasound-guided inferior alveolar nerve block
}

\author{
Sho Kumita1, Hironobu Ueshima1, Hiroshi Otake1, Michiaki Yamakage² \\ 1 Department of Anesthesiology, Showa University Hospital, Tokyo, Japan \\ 2 Department of Anesthesiology, Sapporo Medical University School of Medicine, Hokkaido, Japan
}

\section{Background}

We previously reported a case of successive pain relief of gnathoplasty with inferior alveolar nerve block (IANB) and maxillary nerve block under the ultrasound-guidance.(1) An inferior alveolar nerve blanches off from a mandibular nerve and runs through the pterygomandibular space (PMS), which is between a masseter and a lateral pterygoid muscle, and contains a maxillary artery. Ultrasound-guided IANB is the compartment and the superficial block, so it could be more reduced the risk of vessel puncture than mandibular nerve block. However, there have been few studies evaluating the spread of local analgesics of IANB. We report a cadaveric study of IANB with dye under the ultrasound-guidance.

\section{Method}

IANB was performed with one Thiel's embalmed cadaver. A high frequency linear probe was placed just below the zygomatic arch and the mouth was kept open to identify masseteric muscles clearly. A block needle was inserted in the out-of-plane technique and $5 \mathrm{~mL}$ of green dye was injected into the PMS (Figure 1), where an inferior alveolar nerve was included. A cadaver was dissected 30 minutes after the injection.
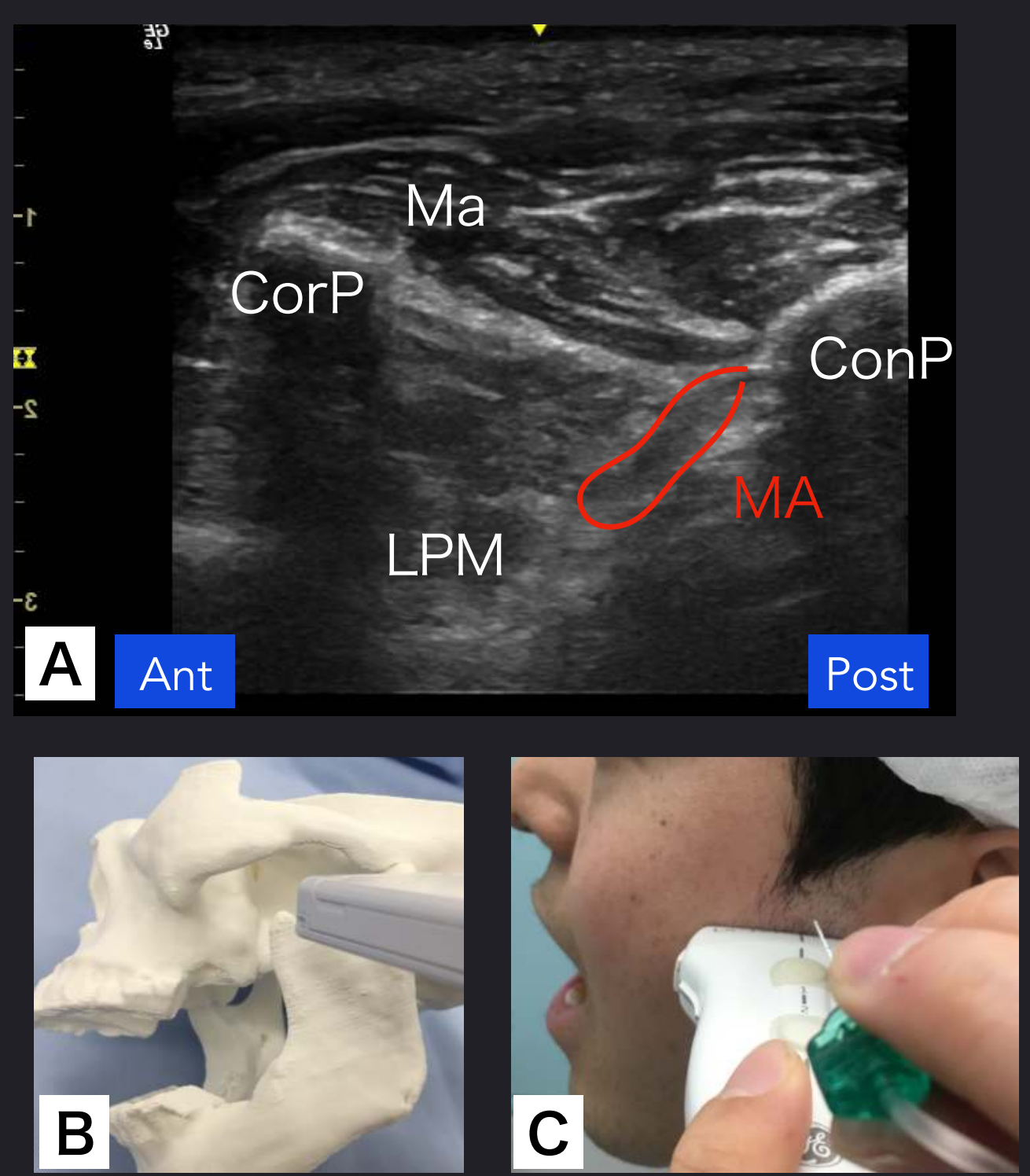

Figure 1. (A) An ultrasound image using a high-frequency linear probe of IANB. $(B, C)$ The position of the probe and the needle of IANB.

Ma, masseter; CorP, coronoid process; ConP, condyle process; LPM, lateral pterygoid muscle; MA, maxillary artery

\section{Result}

The distribution of the dye is shown in Figure 2. The dye was successfully spread into the PMS, and around the inferior alveolar nerve and the lingual nerve. No incidence of injection into the maxillary artery was observed. Unfortunately, the inferior alveolar nerve was injured at the dissection.

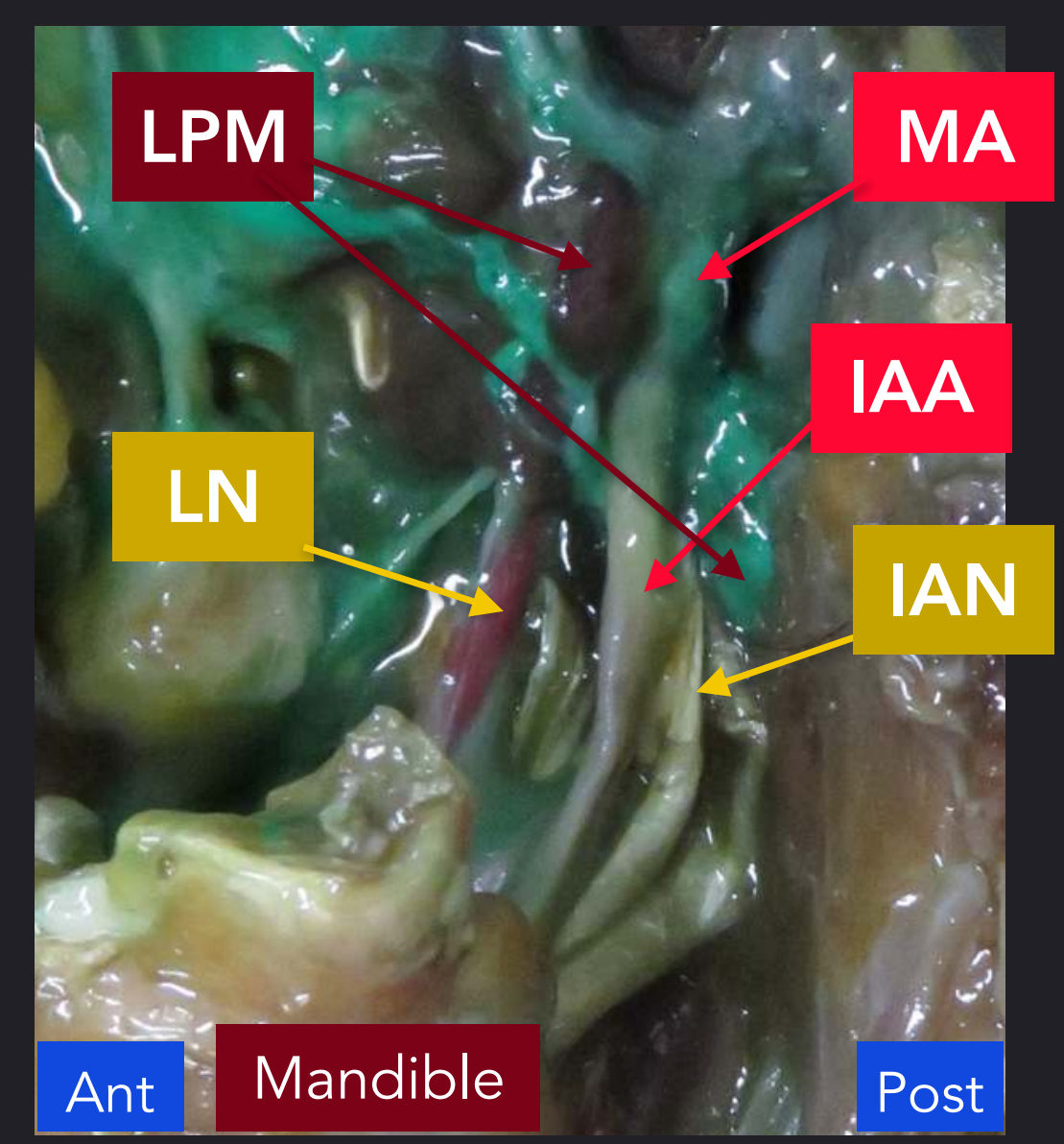

Figure 2. Cadaveric specimen following IANB. Green dye staining can be seen to the inferior alveolar nerve.

LPM, lateral pterygoid muscle; MA, maxillary artery; LN, lingual nerve; IAN, inferior alveolar nerve; IAA, inferior alveolar artery

\section{Discussion}

An inferior alveolar nerve is within the PMS, which is narrow gap and contains mostly loose areolar tissue.(2) Anatomical failures can lead to anesthetic failures of IANB in oral approach.(3) However, ultrasound could visualize the PMS certainly, so that ultrasound-guided IANB may be more effective than into oral approach.

\section{Conclusion}

The present cadaveric study shows that the dye injected into the PMS could spread to the inferior alveolar nerve, and suggests that ultrasound-guided IANB can provide effective analgesia.

\section{References}

(1) Asian J Anesthesia. 2017; 55: 89-90

(2) Australian Dental Journal 2011; 56: 112-121

(3) Folia Medica Cracoviensia Vol. LIII, 1, 2013: 79-85 